\title{
Combined fit of UHECR spectrum and composition with two extragalactic source populations
}

\author{
Saikat Das, ${ }^{a, *}$ Soebur Razzaque ${ }^{b}$ and Nayantara Gupta ${ }^{a}$ \\ ${ }^{a}$ Astronomy \& Astrophysics Group, Raman Research Institute, Bengaluru 560080, Karnataka, India \\ ${ }^{b}$ Centre for Astro-Particle Physics (CAPP) and Department of Physics, University of Johannesburg, P.O. \\ Box 524, Auckland Park 2006, South Africa \\ E-mail: saikatdas@rri.res.in
}

A mixed composition of light-to-heavy nuclei elements $\left({ }^{1} \mathrm{H},{ }^{4} \mathrm{He},{ }^{14} \mathrm{~N},{ }^{28} \mathrm{Si},{ }^{56} \mathrm{Fe}\right)$ at injection fits the ultrahigh-energy cosmic ray (UHECR; $E>10^{17} \mathrm{eV}$ ) spectrum data measured by the Pierre Auger Observatory, beyond the ankle, i.e., $E \gtrsim 5 \times 10^{18} \mathrm{eV}$. However, the composition fit can be further improved by the addition of light nuclei at the highest energies. We consider the light nuclei to originate from a discrete source population consisting of protons $\left({ }^{1} \mathrm{H}\right)$. We constrain the maximum allowed proton fraction at the highest-energy bin at $3.5 \sigma$ statistical significance. Including the redshift evolution of sources as a free parameter further improves the composition fit. We find that low-luminosity gamma-ray bursts match the best-fit evolution index in the case of the one-population model. Active galactic nuclei are the plausible candidates for light nuclei injection in the two-population model, whereas tidal disruption events can inject heavy nuclei composition. We also present the secondary neutrino flux in one- and two-population models, constraining the composition at highest energies.

$37^{\text {th }}$ International Cosmic Ray Conference (ICRC 2021)

July 12th - 23rd, 2021

Online - Berlin, Germany

\footnotetext{
${ }^{*}$ Presenter
} 


\section{Introduction}

The cosmic accelerators of ultrahigh-energy cosmic rays are yet to be discovered. The Pierre Auger Observatory (PAO) in Malargüe, Argentina [1] and the Telescope Array (TA) experiment in Utah, United States [2] are the leading experiments observing UHECR spectrum, composition and anisotropy. Active Galactic Nuclei (AGNs) [3, 4], blazars [5, 6], transient sources like gamma-ray bursts (GRBs) [7], tidal disruption events (TDEs) [8], pulsar winds [9] are potential candidate sources. Recently, PAO has found a $4 \sigma$ correlation between starburst galaxies and the observed intermediate scale anisotropy [10]. The propagation of UHECRs yields secondary neutrino and $\gamma$-rays, which are important messengers of UHECR acceleration. Current multi-messenger observations and also the composition measurement by PAO disfavors a pure proton composition at the highest energies [11, 12]. UHECR induces extensive air shower (EAS) in the Earth's atmosphere, which is reconstructed to measure the maximum shower-depth distribution $\left(X_{\max }\right)$ [13]. The first two moments of $X_{\max }$, i.e., the mean $\left\langle X_{\max }\right\rangle$, and its fluctuation from shower-to-shower $\sigma\left(X_{\max }\right)$ gives the mass composition of incident UHECR particle.

The mass composition is not well known due to uncertainties in hadronic interaction models and hence in the shower propagation codes, eg., CORSIKA, CONEX, etc. The proton fraction decreases with increasing energy above $10^{18.3} \mathrm{eV}$ and ${ }^{14} \mathrm{~N}$ dominates at $10^{19.6} \mathrm{eV}$ for SYBILL2.3c [14] and EPOS-LHC [15] hadronic models. QGSJET-II.04 [16] model shows a ${ }^{4} \mathrm{He}$ dominance at the highest energies. The ankle is interpreted as a transition between two (or more) different source populations and often from Galactic to extragalactic sources. We analyze the combined fit of the UHECR spectrum and mass composition above the ankle in light of two source populations. The extra proton component $\left({ }^{1} \mathrm{H}\right)$ significantly improves the composition fit at the highest energies. The other population injects ${ }^{4} \mathrm{He},{ }^{14} \mathrm{~N},{ }^{28} \mathrm{Si},{ }^{56} \mathrm{Fe}$. The UHECR source parameters for both populations are varied simultaneously to obtain the best-fit case compared to the one-population model. We also calculate the diffuse neutrino flux for both cases, constrained by the upper limits from current detectors [18]. We perform the analysis for a flat source evolution and a redshift-dependent powerlaw evolution of the two populations. The latter case improves the composition fit further. We identify the candidate source populations from the best-fit evolution indices.

\section{UHECR propagation and shower depth distribution}

UHECRs interact with the cosmic microwave background (CMB) and the extragalactic background light (EBL) to undergo photopion production, pair production, producing secondary $\mathrm{e}^{ \pm}$, $\gamma$-rays and neutrinos. Heavier nuclei also undergo photo-disintegration, which is the most efficient energy loss process for them. Nuclear $\beta$-decay also produces secondary neutrinos. All particles are affected by the adiabatic energy losses due to the universe's expansion. We use the public simulation code CRPropa 3 to propagate UHECRs from their sources to Earth and store the secondary particles produced therein [19]. The best-fit UHECR source parameters are calculated by using a general $\chi^{2}$ formalism for the goodness of fit that takes into account both spectrum and composition measured by PAO [20,21] above the ankle $E \gtrsim 5 \cdot 10^{18} \mathrm{eV}$,

$$
\chi_{j}^{2}=\sum_{i=1}^{N}\left[\frac{y_{i}^{\mathrm{obs}}(E)-y_{i}^{\bmod }\left(E ; a_{M}\right)}{\sigma_{i}}\right]^{2}
$$


where the subscript $j$ runs over the three observables, viz., spectrum $E^{2} J(E), X_{\max }$, and $\sigma\left(X_{\max }\right)$. Here $y_{i}^{\mathrm{obs}}(E)$ is the measured value of an observable in the $i$-th energy bin corresponding to a mean energy $E$ and $y_{i}^{\bmod }\left(E ; a_{M}\right)$ is the value obtained numerically. $a_{M}$ are the best-fit values of $M$ parameters varied in the simulations. $\sigma_{i}$ are the errors provided by PAO. We consider that all elements are injected by the sources following the spectrum given as

$$
\frac{d N}{d E}=A_{0} \sum_{i} K_{i}\left(\frac{E}{E_{0}}\right)^{-\alpha} \times \begin{cases}1 & \left(E \leqslant Z R_{\text {cut }}\right) \\ \exp \left(1-\frac{E}{Z R_{\text {cut }}}\right) & \left(E>Z R_{\text {cut }}\right)\end{cases}
$$

$K_{i}, \alpha$, and $R_{\text {cut }}$ are the abundance fraction of elements, spectral index at injection, and the cutoff rigidity, respectively. $A_{0}$ and $E_{0}$ are arbitrary normalization flux and reference energy, respectively. We use the EBL model by Gilmore et al. [22] and TALYS-1.8 photodisintegration cross-section.

We use the parametrizations given by PAO to calculate the mean depth of cosmic-ray air shower maximum $\left\langle X_{\max }\right\rangle$ and its dispersion from the first two moments of $\ln A$ [23].

$$
\begin{aligned}
\left\langle X_{\max }\right\rangle & =\left\langle X_{\max }\right\rangle_{p}+f_{E}\langle\ln A\rangle \\
\sigma^{2}\left(X_{\max }\right) & =\left\langle\sigma_{\text {sh }}^{2}\right\rangle+f_{E}^{2} \sigma_{\ln A}^{2}
\end{aligned}
$$

where $\left\langle X_{\max }\right\rangle_{p}$ is the mean maximum depth of proton showers, and $f_{E}$ is a parameter that depends on the energy of the UHECR event,

$$
f_{E}=\xi-\frac{D}{\ln 10}+\delta \log _{10}\left(\frac{E}{E_{0}}\right)
$$

where $\xi, D$, and $\delta$ depend on the specific hadronic interaction model. $\sigma_{\ln A}^{2}$ is the variance of $\ln A$ distribution and $\left\langle\sigma_{\mathrm{sh}}^{2}\right\rangle$ is the average variance of $X_{\max }$ weighted according to the $\ln A$ distribution,

$$
\left\langle\sigma_{\mathrm{sh}}^{2}\right\rangle=\sigma_{p}^{2}\left[1+a\langle\ln A\rangle+b\left\langle(\ln A)^{2}\right\rangle\right]
$$

where $\sigma_{p}^{2}$ is the $X_{\max }$ variance for proton showers depending on energy and three model-dependent parameters. In this work, we use the updated parameter values ${ }^{1}$ obtained from the CONEX simulations, for one of the post-LHC hadronic interaction models, SYBILL2.3c.

\section{Results}

\subsection{One-population model}

To study the one-population model, we consider a uniform distribution of sources up to a redshift $z=1$ and injecting the stable elements $\mathrm{H}, \mathrm{He}, \mathrm{N}, \mathrm{Si}$, Fe between $0.1-1000 \mathrm{EeV}$, following Eq. 2. The cutoff in the spectrum is dominated by ${ }^{56} \mathrm{Fe}$, resulting from the maximum acceleration energy at the sources. We scan the parameter space by varying the rigidity cutoff $\log _{10}\left(R_{\text {cut }} / \mathrm{V}\right)$ between [18.0, 18.5] with a grid spacing of 0.1 and the injection spectral index $\alpha$ between $[-1.5,1.0]$ with a grid spacing of 0.1 . For each set of values $\left\{\alpha, \log _{10}\left(R_{\text {cut }} / \mathrm{V}\right)\right\}$, we find the best-fit abundance fraction of the injected elements. The number of physical parameters varied is 7 , and we consider

${ }^{1}$ S. Petrera and F. Salamida (2018), Pierre Auger Observatory 

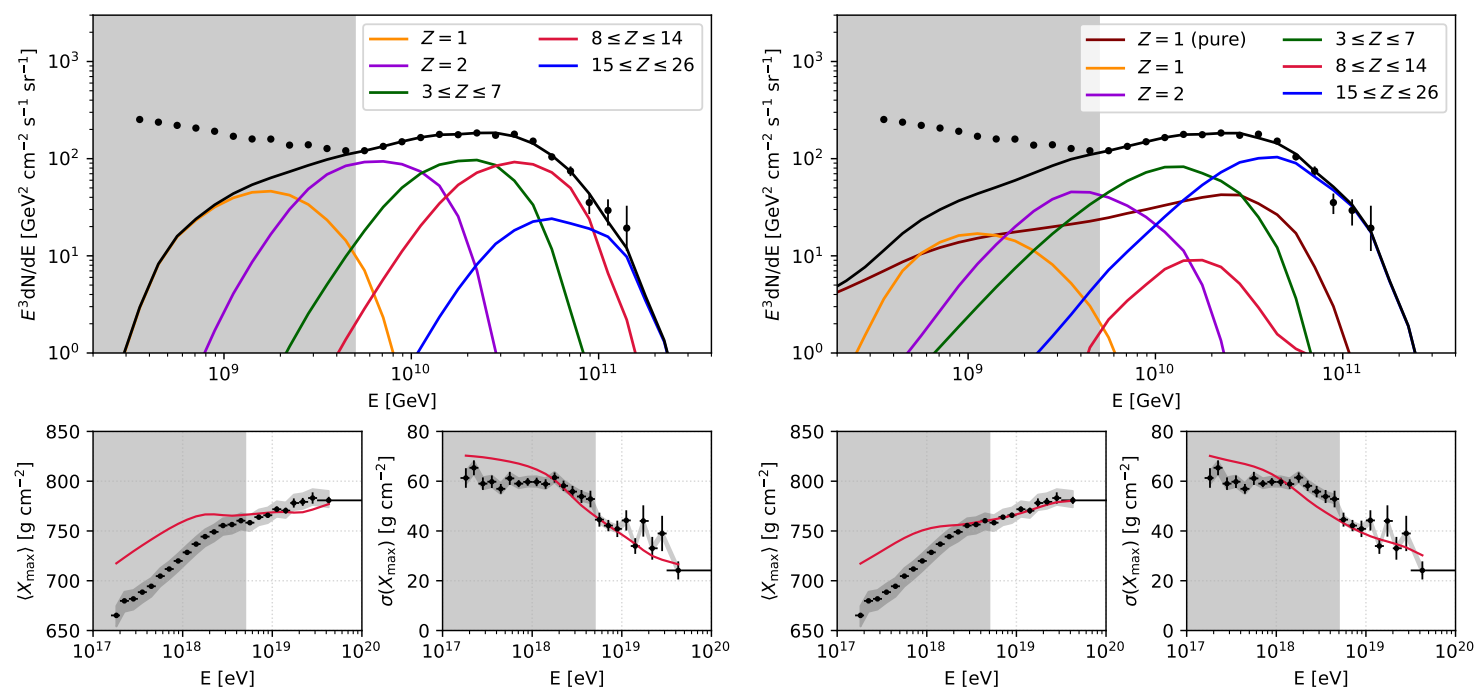

Figure 1: UHECR spectrum and composition for the best-fit parameters of single-population (left) and two-population model (right, $\alpha_{1}=2.2$ ) for a flat $(m=0)$ cosmological evolution of sources. Figure adapted from Ref. [27].
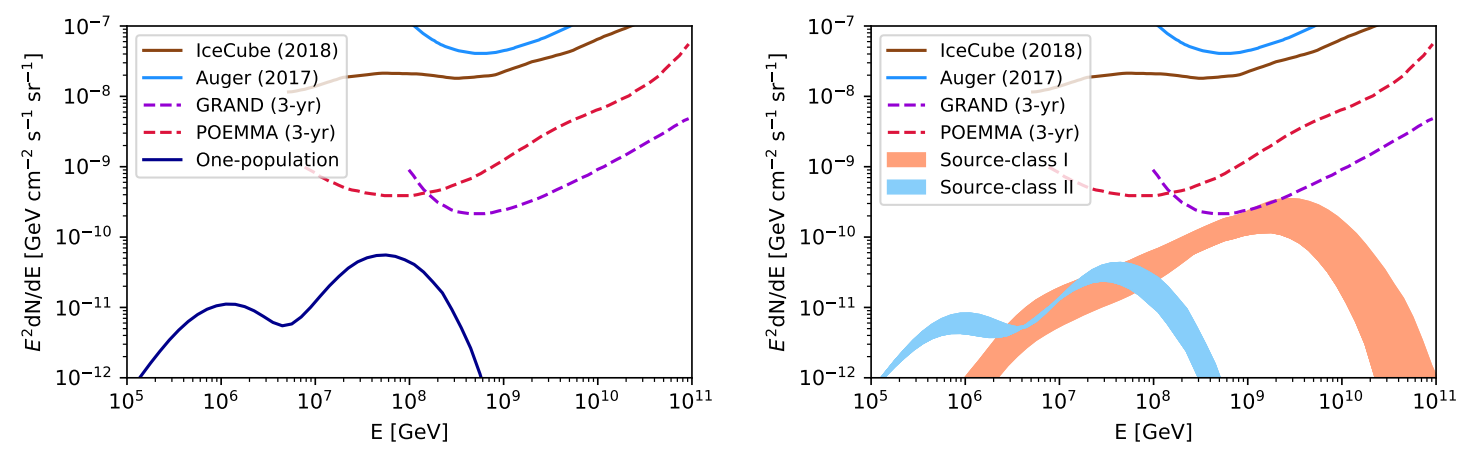

Figure 2: The all-flavor cosmogenic neutrino fluxes for one-population (left) and two-population model (right, $\alpha_{1}=2.2$ ) for a flat $(m=0)$ cosmological evolution of sources. Figure adapted from Ref. [27].

the normalization an additional free parameter. Hence the number of degrees of freedom (d.o.f) is $N_{\mathrm{d}}=33-7-1=25$ in this model since the fitting is done to a total of 33 data points. All the parameter values for the best-fit case of the single-population model are listed in Table 1. We see that the best-fit ${ }^{1} \mathrm{H}$ fraction turns out to be zero, and a non-zero ${ }^{56} \mathrm{Fe}$ component is unavoidable in this case. The best-fit value of the injection spectral index is negative, making it difficult to explain by the Fermi acceleration model. The combined fit by PAO suggests a hard injection spectrum, although positive. The slope of the simulated $X_{\max }$ plot (cf. Fig. 1) suggests that the addition of a light element above $10^{19} \mathrm{eV}$ can improve the fit.

\subsection{Two-population model}

We consider an additional extragalactic source population injecting ${ }^{1} \mathrm{H}$, extending upto the highest-energy bin with rigidity cutoff $R_{\text {cut }, 1}$, and injection spectral index $\alpha_{1} \gtrsim 2$. We refer to 


\begin{tabular}{|c|c|c|c|c|}
\hline Parameter & \multicolumn{3}{|c|}{ Description } & Values \\
\hline$\alpha$ & \multicolumn{3}{|c|}{ Source spectral index } & -0.7 \\
\hline $\log _{10}\left(R_{\text {cut }} / \mathrm{V}\right)$ & \multicolumn{3}{|c|}{ Cutoff rigidity } & $18.2 \mathrm{EV}$ \\
\hline$z_{\max }$ & \multicolumn{3}{|c|}{ Cutoff redshift } & 1.0 (fixed) \\
\hline$m$ & \multicolumn{3}{|c|}{ Source evolution index } & 0.0 (fixed) \\
\hline \multirow[t]{2}{*}{$K_{i}(\%)$} & $\mathrm{H}$ & $\mathrm{He}$ & $\mathrm{N} \quad \mathrm{Si}$ & $\mathrm{Fe}$ \\
\hline & 0.0 & 95.6 & 4.10. & 0.0073 \\
\hline$\chi_{\text {tot }}^{2} /$ d.o.f & $\chi_{\text {spec }}^{2}$ & $\chi_{\text {comp }}^{2}$ & & \\
\hline $56.19 / 25$ & 9.94 & 46.25 & & \\
\hline
\end{tabular}

Table 1: UHECR best-fit parameter set for the one-population model (flat evolution, $m=0$ ). Please see Ref. [27] for more details.

\begin{tabular}{ccc|cccccc|cc}
\hline \multicolumn{3}{c|}{ Source-class I } & \multicolumn{5}{c|}{ Source-class II } & \multicolumn{2}{c}{ Goodness-of-fit } \\
\hline$\alpha_{1}$ & $f_{\mathrm{H}}(\%)$ & $\log _{10}\left(R_{\mathrm{cut}, 1} / \mathrm{V}\right)$ & $\alpha_{2}$ & $\log _{10}\left(R_{\mathrm{cut}, 2} / \mathrm{V}\right)$ & $K_{H e}$ & $K_{N}$ & $K_{S i}$ & $K_{F e}$ & $\chi_{\text {comp }}^{2}$ & $\chi_{\text {tot }}^{2}$ \\
\hline 2.2 & $1.5 \%$ & 19.5 & 0.9 & 18.30 & 53.00 & 44.25 & 0.00 & 2.75 & 15.52 & 30.20 \\
2.4 & $2.5 \%$ & 19.6 & 0.9 & 18.30 & 45.25 & 51.00 & 0.75 & 3.00 & 18.13 & 30.73 \\
2.6 & $2.0 \%$ & 19.6 & 1.1 & 18.30 & 0.00 & 91.50 & 0.00 & 8.50 & 19.63 & 31.66 \\
\hline
\end{tabular}

Table 2: Best-fits to UHECR spectrum and composition for two-population model without cosmological evolution. Please see Ref. [27] for more details.

this as the source-class I (abbv. Cls-I). The normalization $A_{1}=A_{p}$ is fixed by the condition $J_{p}\left(E_{h}\right)=f_{\mathrm{H}} J\left(E_{h}\right)$, where $J(E)=d N / d E$ of the observed spectrum and $E_{h}$ is the mean energy of the highest-energy bin. $f_{\mathrm{H}}$ is an additional parameter that takes care of the proton fraction in the highest-energy bin of the UHECR spectrum. Another population (source-class II, abbv. Cls-II) injects light-to-heavy nuclei, viz., $\mathrm{He}, \mathrm{N}, \mathrm{Si}$, and $\mathrm{Fe}$ with rigidity cutoff $R_{\mathrm{cut}, 2}$, injection spectral index $\alpha_{2}$, and the abundance fraction at injection $K_{i}\left(\sum_{i} K_{i}=100 \%\right)$. The normalization $A_{2}$ in this case is a free parameter which is adjusted to fit the spectrum and composition. We set the maximum redshift of the sources to $z_{\max }=1$ devoid of redshift evolution, i.e., $m=0$ in the $(1+z)^{m}$ type of source evolution models. We vary $f_{\mathrm{H}}$ from $1.0-20.0 \%$, at intervals of $0.5 \%$ between $1.0-2.5 \%$ and at intervals of $2.5 \%$ between $2.5-20.0 \%$. The spectral index $\alpha_{1}$ is varied through the values $2.2,2.4$, and 2.6, inspired by previous analyses with light elements fitting the UHECR spectrum [24]. We vary $\log _{10}\left(R_{\text {cut }, 1} / \mathrm{V}\right)$ between the interval [19.5, 20.2] at grid spacings of 0.1, and $\log _{10}\left(R_{\text {cut }, 2} / \mathrm{V}\right)$ between $[18.22,18.36]$ at grid spacing of 0.02 .

For each combination of $\left\{\alpha_{1}, f_{\mathrm{H}}\right\}$, we find the best-fit values of $\log _{10}\left(R_{\mathrm{cut}, 1} / \mathrm{V}\right), \log _{10}\left(R_{\mathrm{cut}, 2} / \mathrm{V}\right)$, $\alpha_{2}$, and composition $K_{i}$ at injection of Cls-II; that minimizes the $\chi_{\text {tot }}^{2}$ of the combined fit. The best-fits are found at $f_{\mathrm{H}}=1.5 \%, 2.5 \%$, and 2.0\%, respectively for $\alpha_{1}=2.2,2.4$, and 2.6. The corresponding parameter sets are shown in Table 2. A significant improvement in the combined fit is evident for all the cases compared to the one-population model. The top right panel of Fig. 1 shows the best-fit case for $\alpha_{1}=2.2$. The pure-proton component favors higher values of cutoff rigidity than $\mathrm{Cls}$-II and steeper injection spectral index. The all-flavor neutrino fluxes resulting from the one population and two-population models are shown in the left and right panel of Fig. 2. 
The current $90 \%$ C.L. differential flux upper limits imposed by 9-years of IceCube data [18], the current sensitivity by PAO [25] and that predicted for 3-years of observation by GRAND [26] are also overplotted. The hard spectral index and lower maximum rigidity in the one-population model lead to a neutrino spectrum much lower than the current and upcoming neutrino detector sensitivities. The shaded region corresponds to the allowed range of neutrino flux from $\mathrm{Cls}-\mathrm{I}$ and Cls-II in the two-population model for $f_{\mathrm{H}}=1.0-20.0 \%$. With further increase in exposure time, GRAND should constrain our two-population model parameters if $f_{H} \gtrsim 10 \%$.

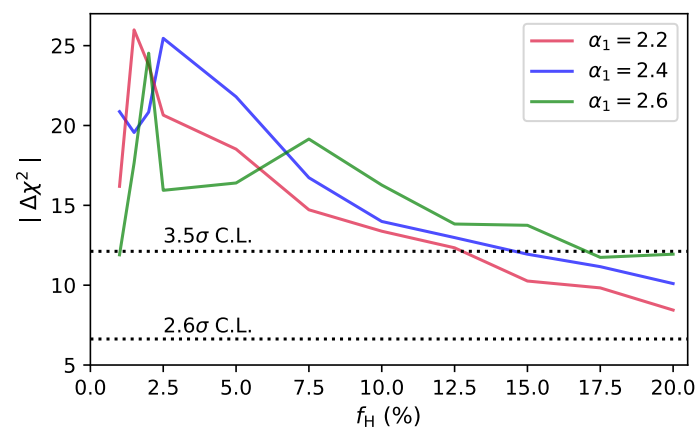

Figure 3: $\left|\Delta \chi^{2}\right|$ values between the one-population and two-population model (without cosmological evolutions) for one d.o.f are shown as a function of the pure-proton fraction $f_{\mathrm{H}}$. Three lines correspond to three values of $\mathrm{Cls}-\mathrm{I}$ injection spectral index. Figure adapted from Ref. [27]

Since the best-fit $\mathrm{H}$ fraction is zero in Table-I, $K_{\mathrm{H}}$ is a redundant parameter, the actual number of parameters is six, coinciding with that of $\mathrm{Cls}$-II in Table-II. Hence, the difference in the number of parameters varied between one-population and two-population models is one, i.e., $R_{\text {cut, } 1}$. A smooth transition from the two-population model to one-population model can be done by setting $R_{\mathrm{cut}, 1}=0$. This necessarily implies that $f_{\mathrm{H}}=0$ and there remains no $\alpha_{1}$. Based on the values obtained using

$$
\Delta \chi^{2}=\left.\chi^{2}\right|_{R_{c u t, 1}}-\left.\chi^{2}\right|_{R_{\text {cut }, 1}=0}
$$

we estimate the maximum allowed proton fraction at $3.5 \sigma$ confidence level (C.L.) in the highestenergy bin. For $\alpha_{1}=2.2$ this corresponds to $\approx 12.5 \%, \alpha_{1}=2.4$ corresponds to $\approx 15.0 \%$, and for $\alpha_{1}=2.6$ it turns out to be $\approx 17.5 \%$. However the maximum $\left|\Delta \chi^{2}\right|$ is found for $\alpha_{1}=2.2$ indicating the most significant improvement, as shown in Fig. 3. A similar analysis can be done considering a power-law evolution of the sources in redshift, given as $(1+z)^{m}$, where $m$ is taken to be discrete for each population. The best-fit obtained for $m_{1}, m_{2} \neq 0$ is better than the flat evolution case and compared to the one-population model with $m \neq 0$. Please refer to [27] for a detailed analysis with redshift evolution, including the correlation between fit parameters and identification of candidate sources for $\mathrm{Cls}-\mathrm{I}$ and $\mathrm{Cls}$-II.

\section{Discussions and Conclusions}

The simulated $\sigma\left(X_{\max }\right)$ in the one-population model indicates that the addition of a light nuclei component up to the highest observed energies can improve the combined fit of the UHECR spectrum and composition. We explore a two-population model where the additional source class 
injects ${ }^{1} \mathrm{H}$ extending up to the highest observed energies. Our fit region excludes the energy range below the ankle and aims at improving the composition fit at the highest energies. We do not assume any fixed abundance fraction for the light-to-heavy nuclei injecting sources and calculate the best-fit values by simultaneously fitting the composition data $X_{\max }, \sigma\left(X_{\max }\right)$, and the energy spectrum. Here, we find a significant improvement in the combined fit to spectrum and composition data exceeding $\gtrsim 3 \sigma$ in some cases. A non-zero proton fraction is inevitable. Although a positive evolution index is preferred in the one-population model, the best-fit value changes sign in the two-population model. Only the sources within $z \lesssim 1$ are considered because UHECRs from higher redshift contribute below the ankle due to increased photodisintegration.

The cumulative neutrino spectrum in the two-population model at $E \gtrsim 0.1 \mathrm{EeV}$ is dominated by GZK neutrinos from $p \gamma_{\mathrm{CMB}}$ interactions due to the high values of $E_{\max }$ for Cls-I near the delta-resonance threshold. The double-humped feature of the neutrino spectrum is a signature of interactions on the $\mathrm{CMB}$ and EBL by cosmic rays of different energies. The higher energy peak at $\sim 3 \cdot 10^{18} \mathrm{eV}$ resulting from proton primaries will be a robust test of the presence of a light component at the highest energies. Higher values of $z_{\max }$ and a variation of $m$ can significantly affect the neutrino spectrum. Thus, within this model's minimal requirements, our neutrino spectrum can be considered as a conservative lower bound in the two-population scenario. The flux of secondary photons increases with an increasing value of $\alpha_{1}$. The cosmogenic photon spectrum in proton-dip model saturates the diffuse gamma-ray background at $\sim 1 \mathrm{TeV}$ for $\alpha_{1}=2.6, m=0$ [24]. In our two-population model, the proton fraction is much lower at the highest energies. Thus the secondary photons from Cls-I are well within the upper bound imposed by Fermi-LAT [28]. For Cls-II injecting heavier nuclei contributes weakly to the cosmogenic photon flux.

An analysis including the redshift evolution of source classes as a free parameter is presented in details in Ref. [27]. Luminous AGNs and GRBs match the evolution index required for Cls-I, accelerating protons to ultrahigh energies. Furthermore, the high negative redshift evolution index and substantial Fe fraction allow us to identify the Cls-II with tidal disruption events (TDEs).

\section{References}

[1] A. Aab et al. (Pierre Auger Collaboration), Nucl. Instrum. Meth. A 798 (2015), 172-213 [arXiv: 1502.01323].

[2] R. U. Abbasi et al. (Telescope Array Collaboration), Astropart. Phys. 80 (2016), 131-140 [arXiv: 1511.07510].

[3] C. D. Dermer, S. Razzaque, J. D. Finke and A. Atoyan, New J. Phys. 11 (2009), 065016 [arXiv:0811.1160].

[4] X. Wang and A. Loeb, Phys. Rev. D 95 (2017) no.6, 063007 [arXiv:1611.07616].

[5] W. Essey and A. Kusenko, Astropart. Phys. 33 (2010), 81-85 [arXiv:0905.1162].

[6] E. Resconi, S. Coenders, P. Padovani, P. Giommi and L. Caccianiga, Mon. Not. Roy. Astron. Soc. 468 (2017) no.1, 597-606 [arXiv:1611.06022]. 
[7] N. Globus, D. Allard, R. Mochkovitch and E. Parizot, Mon. Not. Roy. Astron. Soc. 451 (2015) no.1, 751-790 [arXiv:1409.1271].

[8] G. R. Farrar and T. Piran, [arXiv:1411.0704].

[9] K. Kotera, E. Amato and P. Blasi, JCAP 08 (2015), 026 [arXiv:1503.07907].

[10] A. Aab et al. (Pierre Auger Collaboration), Astrophys. J. Lett. 853 (2018) no.2, L29 [arXiv:1801.06160].

[11] K. Fang and K. Kotera, Astrophys. J. Lett. 832 (2016) no.1, L17 [arXiv:1610.08055].

[12] A. D. Supanitsky, Phys. Rev. D 94 (2016) no.6, 063002 [arXiv:1607.00290].

[13] R. U. Abbasi et al. (Telescope Array Collaboration), Astrophys. J. 862 (2018) no.2, 91 [arXiv:1802.05003].

[14] F. Riehn, R. Engel, A. Fedynitch, T. K. Gaisser and T. Stanev, PoS ICRC2015 (2016), 558 [arXiv:1510.00568].

[15] T. Pierog, I. Karpenko, J. M. Katzy, E. Yatsenko and K. Werner, Phys. Rev. C 92 (2015) no.3, 034906 [arXiv:1306.0121].

[16] S. Ostapchenko, Phys. Rev. D 83 (2011), 014018 [arXiv:1010.1869].

[17] M. Unger, G. R. Farrar and L. A. Anchordoqui, Phys. Rev. D 92 (2015) no.12, 123001 [arXiv:1505.02153].

[18] M. G. Aartsen et al. (IceCube Collaboration), Phys. Rev. D 98 (2018) no.6, 062003 [arXiv:1807.01820].

[19] R. Alves Batista et al. JCAP 05 (2016), 038 [arXiv:1603.07142].

[20] F. Fenu (Pierre Auger Collaboration), PoS ICRC2017 (2018), 486

[21] J. Bellido (Pierre Auger Collaboration), PoS ICRC2017 (2018), 506

[22] R. C. Gilmore, R. S. Somerville, J. R. Primack and A. Dominguez, Mon. Not. Roy. Astron. Soc. 422 (2012), 3189 [arXiv:1104.0671].

[23] P. Abreu et al. (Pierre Auger Collaboration), JCAP 02 (2013), 026 [arXiv:1301.6637].

[24] S. Das, S. Razzaque and N. Gupta, Phys. Rev. D 99 (2019) no.8, 083015 [arXiv:1809.05321].

[25] A. Aab et al. (Pierre Auger Collaboration), Phys. Rev. D 91 (2015) no.9, 092008 [arXiv: 1504.05397].

[26] O. Martineau-Huynh et al. (GRAND Collaboration), EPJ Web Conf. 135 (2017), 02001 [arXiv: 1702.01395].

[27] S. Das, S. Razzaque and N. Gupta, Eur. Phys. J. C 81 (2021) no.1, 59 [arXiv:2004.07621].

[28] M. Ackermann et al. (Fermi-LAT collaboration), Astrophys. J. 799 (2015), 86 [arXiv:1410.3696]. 